\title{
T-cell-associated immunotherapy: a promising strategy for the treatment of hepatocellular carcinoma
}

\author{
"Decades of improvement have accelerated the \\ perfection of T-cell immunotherapy. Given the \\ rapid developments in this field, T-cell immunotherapy \\ could be more mature, safe and effective."
}

First draft submitted: 3 May 2017; Accepted for publication: 10 May 2017; Published online: 9 June 2017

Keywords: hepatocellular carcinoma • T cellular immunotherapies - tumor-associated antigen

Hepatocellular carcinoma (HCC) ranks the sixth most common cancer and the third most common cause of cancer mortality worldwide; it has poor prognosis and limited therapeutic options $[1,2]$. The progress gained from knowledge of the association between HCC and the immune system has yielded significant treatment strategy breakthroughs. The loss of tumor-associated antigens (TAAs) or decreased MHC antigen expression, inactivation of T cells by reduced TCR signaling or IL-10 and TGF- $\beta$-mediated suppression, eventually cause a scene of immune tolerance and inactivate tumor-specific $T$ cells that immune system cannot recognize TAAs and arm effective immune response against tumors [3]. T cells are good candidates for tumor immunotherapy to overcome these obstacles and rekindle the compromised immune response of the patient against the tumors. The frequencies of $\mathrm{CD}^{+} \mathrm{T}, \mathrm{CD}^{+}$ cytotoxic T cells, NK, Treg cells in the tumor tissue of HCC are significantly associated with survival. Among patients diagnosed with $\mathrm{HCC}, \mathrm{CD}^{+} \mathrm{T}$ cells respond to HCCspecific TAAs such as AFP, GPC3, MAGEA1 and NY-ESO-1, which can be applied to distinguishing tumor cells from normal tissues [4]. HCC is correlated with persistent $\mathrm{HBV}$ and $\mathrm{HCV}$ infections, viral proteins of $\mathrm{HBV}$ and $\mathrm{HCV}$ could also play a role of TAA in HCC [5]. Through RNA-sequencing and immunohistochemistry data, HCC-specific genes have been identified and validated.

As an emerging method of tumor clearance aiming to afford more effective and selective targeting of tumor cells by recruiting or facilitating the existing tumor-specific immune response, T-cell immunotherapy circumvents many immune evasion mechanisms. Various tumor immunotherapy techniques associated with $T$ cells have been developed during the last two decades and brought excellent outcomes.

Lymphokine-activated killer (LAK) cells therapy could significantly prolong the survival time of HCC patients but failed to decrease the tumor mass [6]. Not only the limited efficacy, but also more seriously, the severe side effects simultaneously owing to the dose of rIL-2 required led to increasing doubts and fading enthusiasm for LAK cells. With the ability of recognizing the TAAs, autologous tumor-infiltrating lymphocytes in HCC patients following tumor resection could be successfully performed with low toxicity. However, these TAA-specific T cells naturally existed in HCC tissues with restricted antitumor ability [7]. The process of isolating tumor-infiltrating lymphocytes from HCC patients and the proliferation in vitro was challenging and only patients in good status could endure rigorous lymphodepletion- and IL-2-based treatments. Compared with LAK

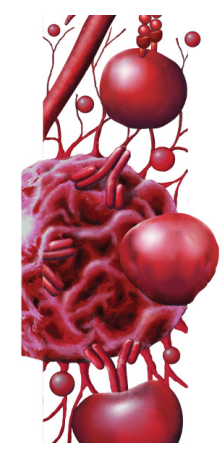

Weijie Ma', Xi Chen ${ }^{1}$ \& Yufeng Yuan*,1 'Department of Hepatobiliary \& Pancreatic Surgery, Zhongnan Hospital of Wuhan University, Wuhan 430071, China *Author for correspondence: yuanyf1971@whu.edu.cn 
cells, cytokine-induced killer (CIK) cells have the feature of high-proliferation rate, potent antitumor effects and minimal cytotoxicity to normal cells. The combined treatment of transcatheter arterial chemoembolization and radiofrequency ablation with sequential CIK immunotherapy could lower the recurrence rate of HCC and prolong the recurrence-free survival and overall survival of HCC patients without severe adverse effects $[8,9]$. Although adverse reactions, like pyrexia, chills, myalgia and fatigue were correlated to CIK cell agents in $17 \%$ of patients, they were not severe enough to delay or cease the therapy [10].

Cytotoxic T lymphocytes (CTLs) are $\mathrm{CD}^{+} \alpha \beta$ $T$ cells which could recognize MHC molecules, present TAAs and release granzyme B and perforin to lyse tumor cells. However, the lack of MHC, the existence of suppressor immune cells, like Tregs, myeloidderived suppressor cells and the shortage of costimulatory molecules restrained the effect of reinfused CTLs. Peptide vaccines similar to TAAs have been attempted to stimulate $T$ cells, causing the proliferation of effective CTLs to kill TAA-positive target cells. Besides, the immune checkpoint blockade is another way. The blocking antibodies of CTLA- 4 and PD- 1 that disrupt CTLA-4/PD-1 pathways aiming to reversing exhausted $\mathrm{T}$ cells have already been approved by US FDA for the treatment of malignancies and currently in development in HCC. CTLA- 4 expressed on Tregs and suppressed effector T-cell activation and function [11]. CTLA-4 acts early for tolerance induction, while PD-1 acts late for long-term tolerance maintenance. The drastically increased expression of PD-1 was found on tumor infiltrating $\mathrm{CD}^{+} \mathrm{T}$ cells in HCC and correlated with poorer disease progression and postoperative relapse. Tremelimumab (CP675,206), which blocks the binding of CTLA-4, has been applied on advanced HCC with HCV-related cirrhosis resulting in promising safety profile and antitumor and antiviral effect [12]. The combined treatment of tremelimumab and tumor ablation for advanced HCC patients brought supporting clinical activity and causes expansion of intratumoral $\mathrm{CD}^{+} \mathrm{T}$ cells [13]. PD-1/PD-L1 blockade could increase the immune response of vaccine-induced GPC3-specific CTLs, and therefore enhance the antitumor effects of a GPC3 peptide vaccine for HCC [14].

By genetic engineering, $T$ cells could be endowed with the capacity to be reactive against tumors. When a tumor antigen peptide fragment presented on the tumor cell MHC was encountered by TCR engineered $\mathrm{T}$ cells, the phosphorylation of immunoreceptor tyrosine-based activation motifs happened, following an intracellular signaling cascade causing the release of cytokines and cytotoxic compounds from T cells. TCR redirected HBV-specific T cells derived from peripheral blood mononuclear cells (PBMCs) of chronic HBV and HBV-related HCC patients had the capabilities of recognizing HBV-infected cells and HCC tumor cells expressing viral antigens [15]. These reinfused HBVTCR-T cells obviously decreased the level of HBsAg in the patient, while, unfortunately, no detectable reduction of the volume of the HCC metastasis [16]. Unlike TCRs, chimeric antigen receptors (CARs) armed $\mathrm{T}$ cells could get rid of the restriction of MHC. Connected by single-chain variable regions $(\mathrm{ScFv})$ of monoclonal antibody heavy and light chains that could identify the TAA and T-cell signal transduction zone, CAR-T cells could distinguish an overexpressed tumor cell surface antigen directly. The second- and thirdgeneration CAR-T cells had the advantage of cytokine production and proliferation in vitro, and mediated tumor regression in xenograft models compared with the first-generation CAR-T cells, the main difference among the three generations was the amount of costimulatory molecules. The success of CAR-T cells in treating hematological malignancies spurred interest in their application to solid tumors. GPC3-specific CAR-T cells has been constructed for the treatment in HCC, which showed significant antitumor activity both in vitro and in vivo [17,18]. CAR-T cells specific for HBV envelope proteins localized to liver in mice, which reduced HBV replication with transient liver damage [19]. Unfortunately, most currently defined tumor antigens were TAAs that were not expressed in vital tissues or relatively higher expressed in tumor cells than normal cells. Therefore, CAR-modified T cells might attack the tumor tissues and cause damage to normal tissues or organs at the mean time. The inefficient homing of $\mathrm{T}$ cells to tumor sites, and the immunosuppressive microenvironment of solid tumors are still waiting to be solved.

Derived from the bispecific antibodies composed of two ScFvs, one that recognizes a TAA and another that binds $\mathrm{CD} 3$ on $\mathrm{T}$ cells, bispecific T-cell engagers (BiTEs) could recruit and activate $\mathrm{T}$ cells, which could be largely indispensable for conventional T-cell recognition molecules like MHC class I molecules and costimulatory proteins. The epithelial cell adhesion molecule (Ep-CAM)-specific BiTE (MT110) had the potential to be applied in various human carcinomas. The encouraging results ignited the passion of applying BiTE to treat HCC. Recently, anti-EpCAM BiTE $1 \mathrm{H} 8 / \mathrm{CD} 3$ has been proved a promising therapeutic agent for HCC treatment [20].

Decades of improvement have accelerated the perfection of T-cell immunotherapy. Given the rapid developments in this field, T-cell immunotherapy could be more mature, safe and effective. 


\section{Financial \& competing interests disclosure}

This work was supported by Hubei Province health and family planning scientific research project (WJ2017Z007). The authors have no other relevant affiliations or financial involvement with any organization or entity with a financial interest in or financial conflict with the subject matter or materials discussed in the manuscript apart from those disclosed.

No writing assistance was utilized in the production of this manuscript.

\section{References}

1 Lafaro KJ, Demirjian AN, Pawlik TM. Epidemiology of hepatocellular carcinoma. Surg. Oncol. Clin. N. Am. 24(1), $1-17$ (2015).

2 Fong ZV, Tanabe KK. The clinical management of hepatocellular carcinoma in the United States, Europe, and Asia: a comprehensive and evidence-based comparison and review. Cancer 120(18), 2824-2838 (2014).

3 Marr LA, Gilham DE, Campbell JD, Fraser AR. Immunology in the clinic review series; focus on cancer: double trouble for tumours: bi-functional and redirected $\mathrm{T}$ cells as effective cancer immunotherapies. Clin. Exp. Immunol. 167(2), 216-225 (2012).

4 Flecken T, Schmidt N, Hild S et al. Immunodominance and functional alterations of tumor-associated antigenspecific $\mathrm{CD}^{+}{ }^{+} \mathrm{T}$-cell responses in hepatocellular carcinoma. Hepatology 59(4), 1415-1426 (2014).

5 Ilan Y. Immune therapy for hepatocellular carcinoma. Hepatol. Int. 8(Suppl. 2) 499-504 (2014).

6 Bertelli R, Neri F, Tsivian M et al. Endolymphatic immunotherapy in inoperable hepatocellular carcinoma. Transplant Proc. 40 (6), 1913-1915 (2008).

7 Li KK, Adams DH. Antitumor CD8 ${ }^{+} \mathrm{T}$ cells in hepatocellular carcinoma: present but exhausted. Hepatology 59(4), 1232-1234 (2014)

8 Huang ZM, Li W, Li S et al. Cytokine-induced killer cells in combination with transcatheter arterial chemoembolization and radiofrequency ablation for hepatocellular carcinoma patients. J. Immunother. 36(5), 287-293 (2013).

9 Cui J, Wang N, Zhao $\mathrm{H}$ et al. Combination of radiofrequency ablation and sequential cellular immunotherapy improves progression-free survival for patients with hepatocellular carcinoma. Int. J. Cancer 134(2), 342-351 (2014).
10 Lee JH, Lee JH, Lim YS et al. Adjuvant immunotherapy with autologous cytokine-induced killer cells for hepatocellular carcinoma. Gastroenterology 148(7), 1383-1391 e1386 (2015).

11 Wing K, Onishi Y, Prieto-Martin P et al. CTLA-4 control over Foxp3+ regulatory T cell function. Science 322(5899), 271-275 (2008).

12 Sangro B, Gomez-Martin C, de la Mata M et al. A clinical trial of CTLA- 4 blockade with tremelimumab in patients with hepatocellular carcinoma and chronic hepatitis C. J. Hepatol 59(1), 81-88 (2013).

13 Duffy AG, Ulahannan SV, Makorova-Rusher O et al. Tremelimumab in combination with ablation in patients with advanced hepatocellular carcinoma. J. Hepatol.doi:10.1016/j. jhep.2016.10.029 (2016) (Epub ahead of print).

14 Sawada Y, Yoshikawa T, Shimomura M, Iwama T, Endo I, Nakatsura T. Programmed death-1 blockade enhances the antitumor effects of peptide vaccine-induced peptide-specific cytotoxic T lymphocytes. Int. J. Oncol. 46(1), 28-36 (2015).

15 Gehring AJ, Xue SA, Ho ZZ et al. Engineering virusspecific $\mathrm{T}$ cells that target $\mathrm{HBV}$ infected hepatocytes and hepatocellular carcinoma cell lines. J. Hepatol. 55(1), 103-110 (2011)

16 Bertoletti A, Brunetto M, Maini MK, Bonino F, Qasim W, Stauss H. T cell receptor-therapy in HBV-related hepatocellularcarcinoma. Oncoimmunology 4(6), e1008354 (2015).

17 Gao H, Li K, Tu H et al. Development of T cells redirected to glypican-3 for the treatment of hepatocellular carcinoma. Clin. Cancer Res. 20(24), 6418-6428 (2014).

$18 \mathrm{Li}$ W, Guo L, Rathi P et al. Redirecting T cells to glypican-3 with 4-1BB zeta chimeric antigen receptors results in Th1 polarization and potent antitumor activity. Hum. Gene Ther. doi:10.1089/hum.2016.025 (2016) (Epub ahead of print).

19 Krebs K, Bottinger N, Huang LR et al. T cells expressing a chimeric antigen receptor that binds hepatitis $\mathrm{B}$ virus envelope proteins control virus replication in mice. Gastroenterology 145(2), 456-465 (2013).

20 Zhang P, Shi B, Gao H et al. An EpCAM/CD3 bispecific antibody efficiently eliminates hepatocellular carcinoma cells with limited galectin-1 expression. Cancer Immunol. Immunother. 63(2), 121-132 (2014). 
